\title{
Modeling and Extended State Observer Based Dynamic Surface Control for Cold Rolling Mill System
}

\author{
$\mathrm{Xu} \mathrm{Li}^{1}$ and Xin Zhang ${ }^{2}$ \\ ${ }^{1}$ The State Key Laboratory of Rolling Automation, Northeastern University, Shenyang 110819, China \\ ${ }^{2}$ School of Resources and Materials, Northeastern University, Qinhuangdao Branch, Qinhuangdao 066004, China \\ Correspondence should be addressed to Xu Li; fly2000lixu@163.com
}

Received 23 September 2015; Revised 17 January 2016; Accepted 16 February 2016

Academic Editor: Thierry Floquet

Copyright (c) $2016 \mathrm{X}$. Li and X. Zhang. This is an open access article distributed under the Creative Commons Attribution License, which permits unrestricted use, distribution, and reproduction in any medium, provided the original work is properly cited.

\begin{abstract}
The modeling and control problems are investigated for cold rolling mill system. Firstly, we establish a monitor automatic gauge control (MAGC) model for a practical cold rolling mill system. The new model is with mismatched uncertainties. Then, an extended state observer (ESO) is developed to estimate uncertainties. In the general high-order systems, the ESO is also used to estimate states. By dynamic surface control method, we design the controller to guarantee stabilization of the cold rolling mill system. Furthermore, we extend proposed method to general high-order systems, in which we analyze the difference from cold rolling mill system. Finally, simulation results for MAGC system are presented to demonstrate the effectiveness of the proposed control strategy.
\end{abstract}

\section{Introduction}

Steel strips with different thicknesses are important products in iron and steel industry, which have a wide range of applications in household electric, automobile manufacturing, machinery, and many other fields. High performance estimation and control techniques are needed for cold rolling mills due to increased demands on productivity and products quality [1]. The monitor automatic gauge control (MAGC) system of cold rolling mill is directly related to the quality and effectiveness of cold rolling strips. Thereby how to ensure stability and improve the performance of MAGC system are becoming a hot research topic in metal processing field.

Accuracy of the mathematical model of automatic gauge control system plays a significant role in products quality, shown in $[2,3]$. It is shown in [4] that the automatic gauge control system is a typical complicated nonlinear one, subject to load uncertainties and disturbances. Therefore, the accurate model is difficult to construct for a complicated MAGC system. In this paper, we establish a dynamic mathematical model of the system with unknown nonlinear functions, which reflects the actual system exactly.

As we know, there usually exist uncertainties and disturbances in practical industrial systems and sometimes only partial state variables can be measured for controller design. Therefore, many observer design methods have been proposed in existing literatures [5-9]. Compared with the traditional observer design method, an extended state observer (ESO) can be used to estimate the unknown uncertainties and disturbances, which can be found in [10-12]. As the MAGC model we construct in this paper has mismatched uncertainties, we will design a new ESO to estimate them and extend our method to a general high-order system.

In high-order strict feedback or pure feedback nonlinear systems, we usually use backstepping method to design the controller. At the same time, the problem of "explosion of complexity" is caused by repeated differentiations. With the increase of system order, the controller becomes more and more complex [13-16]. In order to overcome the problem "explosion of complexity", Swaroop et al. [17] proposed a dynamic surface control (DSC) technique by introducing a first-order filtering. So far many researchers have developed this method, such as [18-23]. The work [20] investigated decentralized dynamic surface control for a class of interconnected nonlinear systems. The robust stabilization method is proposed for uncertain nonlinear systems with unknown time delays via the dynamic surface control in [22]. 


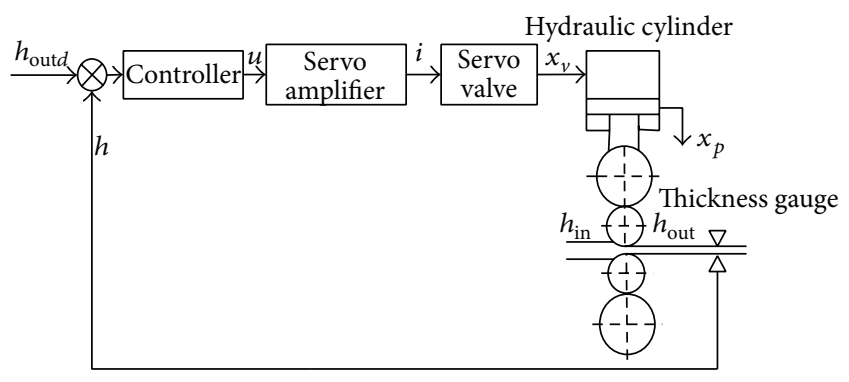

FIGURE 1: Basic configuration of MAGC system.

This paper investigates the dynamic surface control (DSC) problem for MAGC systems, in which the uncertainties are estimated by the ESO. Further, the method is extended to general high-order systems. The contributions of our paper are as follows: (i) The fourth-order system model of the MAGC system is established with uncertainties; (ii) based on the designed extended state observer (ESO) and by dynamic surface control (DSC) method, the uncertainties and the problem "explosion of complexity" are addressed, respectively; (iii) the method is extended to a kind of general high-order systems, and some restrictive conditions are presented. Finally, simulation results are given to demonstrate the effectiveness of the proposed control scheme.

The rest of the paper is organized as follows. Section 2 presents the model of the system. In Section 3, a new ESO is designed to estimate uncertain functions. A DSC method is proposed to design the controller in Section 4. The proposed strategy is extended to general high-order systems in Section 5. Simulation results are illustrated in Section 6 and finally the conclusion is given in Section 7.

\section{Modeling of MAGC System}

MAGC system is a typical electrohydraulic servo system with nonlinear attributes and uncertain properties. It consists of controller, servo amplifier, servo valve, hydraulic cylinder, roller system, body of rolling mill, thickness gauge, and some other sensors [24]. The configuration of MAGC system is shown in Figure 1.

The servo amplifier is a purely proportional part, which can be expressed as

$$
i=K_{p} u
$$

where $K_{p}$ is the amplifier coefficient.

The servo valve treats current $i$ as the input and valve core displacement $x_{v}$ as the output. When the system inherent frequency is below $50 \mathrm{~Hz}$, the relationship can be expressed by

$$
\frac{x_{v}}{i}=\frac{K_{v}}{1+s / \omega_{v}} .
$$

When the inherent frequency is above $50 \mathrm{~Hz}$, it is

$$
\frac{x_{v}}{i}=\frac{K_{v}}{s^{2} / \omega_{v}^{2}+2 \zeta_{v} s / \omega_{v}+1} \text {, }
$$

where $K_{v}$ is the amplifier coefficient of valve core displacement and $\omega_{v}$ and $\zeta_{v}$ are the inherent frequency and damping coefficient of the servo valve, respectively. Because of the fast response speed, it is always regarded as a proportional link

$$
x_{v}=K_{v} i .
$$

Servo valve flux equation is a typical nonlinear loop:

$$
Q_{L}=C_{d} \omega x_{v} \sqrt{\frac{P_{s}-\operatorname{sgn}\left(P_{L}\right)}{\rho}},
$$

where $C_{d}$ is the flux coefficient of the slide valve mouth, $\omega$ is the area grade of the slide valve, $\rho$ is oil density, $P_{s}$ is the inlet pressure of the servo valve, and $P_{L}$ is the outlet pressure of the servo valve.

The flow from servo valve into the cylinder, besides driving the piston movement, can be used to compensate various cylinder leaks and liquid compressed volume, and so forth. The continuous flux equation of the cylinder is

$$
Q_{L}=A_{p} \dot{x}_{p}+C_{t} P_{L}+\frac{V}{4 \beta_{e}} \dot{P}_{L}
$$

where $A_{p}$ is the effective area of the cylinder piston, $x_{p}$ is the cylinder piston displacement, $C_{t}$ is the total leakage coefficient, $\beta_{e}$ is the bulk modulus of elasticity, and $V$ is the cavity volume of the hydraulic cylinder.

The output rolling force of the cylinder keeps balance with the inertia force of the moving parts, viscous damping force, elastic load force, and other load force. The dynamic equation can be written as

$$
A_{p} P_{L}=M_{t} \ddot{x}_{p}+B_{p} \dot{x}_{p}+K_{t} x_{p}+F_{L}
$$

where $M_{t}$ is the equivalent total mass of moving parts of the upper roller system, $B_{p}$ is the viscosity coefficient of cylinder, $K_{t}$ is the elastic stiffness coefficient of load, and $F_{L}$ is other load force acting on the piston, such as the coulomb friction force and rolled piece deformation resistance. And $F_{L}$ can be expressed by

$$
F_{L}=W_{s}\left(h_{\text {in }}-h_{\text {out }}\right)+F_{f},
$$

where $W_{s}$ is the plastic stiffness coefficient of the rolled piece, $F_{f}$ is the unknown force including the coulomb friction force, and $h_{\text {in }}$ and $h_{\text {out }}$ are the input and output thickness of the rolled piece, respectively.

If the elastic recovery of rolled piece is ignored after rolling, the output thickness $h_{\text {out }}$ is equal to the loading roll gap $h_{L}$, and they have dynamic equation

$$
T_{h} \dot{h}_{\text {out }}+h_{\text {out }}=h_{L}
$$

where $T_{h}$ is the inertia time constant.

By ignoring the roll eccentricity, the relationship between the loading roll gap and cylinder displacement is

$$
h_{L}=h_{s}-\Delta h_{r}=h_{s}-\left(x_{p}-\Delta h_{j}\right)=h_{s}-x_{p}+\frac{\Delta F}{M_{p}},
$$


where $h_{s}$ is the set value of roll gap, $\Delta h_{r}$ is the variation quantity of it, $\Delta F$ is the variation quantity of rolling force, and $M_{p}$ is the longitudinal stiffness coefficient of the mill.

Based on the above equations, we choose state variables as $x_{1}=h_{\text {out }}-h_{\text {outd }}, x_{2}=x_{p}-x_{p d}, x_{3}=\dot{x}_{2}, x_{4}=\dot{x}_{3}$, where $h_{\text {outd }}$ and $x_{p d}$ are the desired values of thickness and cylinder piston displacement. Then the state space expression of the system can be expressed as

$$
\begin{aligned}
\dot{x}_{1}= & -\frac{1}{T_{h}} x_{1}-\frac{1}{T_{h}} x_{2}+f_{1}(x, t) \\
\dot{x}_{2}= & x_{3} \\
\dot{x}_{3}= & x_{4} \\
\dot{x}_{4}= & -\left(\frac{W_{s}}{M_{t} T_{h}}-\frac{4 \beta_{e} C_{t} W_{s}}{M_{t} V}\right) x_{1} \\
& -\left(\frac{W_{s}}{M_{t} T_{h}}+\frac{4 \beta_{e} K_{t} C_{t}}{M_{t} V}\right) x_{2} \\
& -\left[\frac{4 \beta_{e}}{M_{t} V}\left(B_{p} C_{t}+A_{p}^{2}\right)+\frac{K_{t}}{M_{t}}\right] x_{3} \\
& -\left(\frac{4 \beta_{e} C_{t}}{V}+\frac{B_{p}}{M_{t}}\right) x_{4} \\
& -\frac{4 \beta_{e} C_{t}}{M_{t} V}\left(K_{t} x_{p d}+W_{s}\left(h_{\mathrm{in}}-h_{\text {out } d}\right)\right) \\
& +\frac{4 \beta_{e} A_{p} K_{v} K_{p}}{M_{t} V} C_{d} \omega \sqrt{\frac{P_{s}-\operatorname{sgn}\left(P_{L}\right)}{\rho}} u(t) \\
& f_{2}(x, t),
\end{aligned}
$$

where $f_{1}(x, t)=\left(1 / T_{h}\right)\left(-x_{p d}+h_{s}-h_{\text {outd }}+\Delta F / M_{p}\right)$ and $f_{2}(x, t)=-\left(1 / M_{t}\right) \dot{F}_{f}-\left(4 \beta_{e} C_{t} / M_{t} V\right) F_{f}+\left(W_{s} / M_{t}\right) f_{1}(x, t)$ are unknown nonlinear terms including the back pressure in hydraulic cylinder, nonlinear characteristics in the servo valve, the friction and elastic deformation force in the rollers, and parameter uncertainties in rolling system, which will be estimated by ESO. For simplicity, choose $a_{11}=1 / T_{h}, a_{41}=W_{s} / M_{t} T_{h}-$ $4 \beta_{e} C_{t} W_{s} / M_{t} V, a_{42}=W_{s} / M_{t} T_{h}+4 \beta_{e} K_{t} C_{t} / M_{t} V, a_{43}=$ $\left(4 \beta_{e} / M_{t} V\right)\left(B_{p} C_{t}+A_{p}^{2}\right)+K_{t} / M_{t}, a_{44}=4 \beta_{e} C_{t} / V+B_{p} / M_{t}$, $v(t)=\left(4 \beta_{e} A_{p} K_{v} K_{p} / M_{t} V\right) C_{d} \omega \sqrt{\left(\left(P_{s}-\operatorname{sgn}\left(P_{L}\right)\right) / \rho\right)} u(t)-$ $\left(4 \beta_{e} C_{t} / M_{t} V\right)\left(K_{t} x_{p d}+W_{s}\left(h_{\mathrm{in}}-h_{\text {out }}\right)\right)$. Because $P_{L}$ can be measured, we design $v(t)$ instead of the controller $u(t)$. Further, system (11) can be written as

$$
\begin{aligned}
& \dot{x}(t)=A x(t)+b_{u}(v+g(x))+B_{d} f(x(t), t), \\
& Y(t)=C x(t),
\end{aligned}
$$

where $g(x)=-a_{41} x_{1}-a_{42} x_{2}-a_{43} x_{3}-a_{44} x_{4}$ and

$$
A=\left[\begin{array}{cccc}
-a_{11} & -a_{11} & 0 & 0 \\
0 & 0 & 1 & 0 \\
0 & 0 & 0 & 1 \\
0 & 0 & 0 & 0
\end{array}\right] \text {, }
$$

$$
\begin{aligned}
b_{u} & =\left[\begin{array}{l}
0 \\
0 \\
0 \\
1
\end{array}\right], \\
B_{d} & =\left[\begin{array}{ll}
1 & 0 \\
0 & 0 \\
0 & 0 \\
0 & 1
\end{array}\right], \\
C & =\left[\begin{array}{lll}
1 & 0 & 0 \\
0 & 1 & 0 \\
0
\end{array}\right], \\
f(x, t) & =\left[\begin{array}{l}
f_{1}(x, t) \\
f_{2}(x, t)
\end{array}\right] .
\end{aligned}
$$

In system (12), function $f(\cdot)$ is not available for the controller design. The control objective is to design an ESO to estimate unknown functions and then using the DSC technique to design a controller $v(t)$ such that outputs and states both converge to a set with an acceptable accuracy. Based on the DSC technique, the proposed control system can eliminate the problem of "explosion of complexity" inherent in the backstepping design method.

Remark 1. In this section, we establish the mathematical modeling for the MAGC system. From (12), we can see that the mathematical model of the system has mismatched uncertainties and is with strict feedback form without considering the term $f(\cdot)$. In order to address the problem of "explosion of complexity," we choose DSC technique to design the controller.

In next section, we will first design the ESO to estimate unknown functions to prepare for the controller design.

\section{Extended State Observer Design}

In general, a nonlinear ESO is always used to get accurate estimation, but it is tanglesome to design. The tandem rolling control system has high speed and needs fast sampling frequency, so we design a linear ESO to meet its simple, practical, and fast-speed demand. In this section, the ESO is only used to estimate the uncertainties and the states of the system are assumed to be measurable.

System (12) has four state variables and two unknown nonlinear functions. We make the two functions as the extended fifth and sixth state variables and $h_{1}, h_{2}$ as their derivatives. Consider

$$
\begin{aligned}
& x_{5}=f_{1}(x, t), \\
& x_{6}=f_{2}(x, t), \\
& h_{1}=\frac{d f_{1}}{d t}, \\
& h_{2}=\frac{d f_{2}}{d t} .
\end{aligned}
$$


By combining (12) with (14), the extended state equation is given by

$$
\begin{aligned}
& \dot{\bar{x}}=\bar{A} \bar{x}+\bar{b}_{u} v_{1}+E h(t), \\
& y=\bar{C} \bar{x},
\end{aligned}
$$

where variables

$$
\begin{aligned}
\bar{x} & =\left[\begin{array}{lll}
x & x_{5} & x_{6}
\end{array}\right]^{T}, \\
h(t) & =\left[\begin{array}{ll}
h_{1} & h_{2}
\end{array}\right]^{T}, \\
v_{1} & =v+g(x)
\end{aligned}
$$

and matrices

$$
\begin{aligned}
\bar{A} & =\left[\begin{array}{cc}
A_{4 \times 4} & \left(B_{d}\right)_{4 \times 2} \\
0_{2 \times 4} & 0_{2 \times 2}
\end{array}\right], \\
\bar{b}_{u} & =\left[\begin{array}{c}
\left(b_{u}\right)_{4 \times 1} \\
0_{2 \times 1}
\end{array}\right], \\
E & =\left[\begin{array}{c}
0_{4 \times 2} \\
I_{2 \times 2}
\end{array}\right], \\
\bar{C} & =\left[\begin{array}{ll}
C_{2 \times 4} & 0_{2 \times 2}
\end{array}\right] .
\end{aligned}
$$

For system (15), we design the ESO as follows:

$$
\begin{aligned}
& \dot{\hat{x}}=\bar{A} \hat{x}+\bar{b}_{u} v_{1}+L(y-\widehat{y}), \\
& \hat{y}=\bar{C} \hat{x},
\end{aligned}
$$

where $\hat{x}$ is the estimate of the state $\bar{x}$ and $L$ is the observer gain matrix with dimension $6 \times 2$.

The extended state error is defined as

$$
\tilde{x}=\bar{x}-\widehat{x}
$$

Subtracting (18) from (15), we get

$$
\dot{\tilde{x}}=\bar{A}_{e} \tilde{x}+E h(t)
$$

where $\bar{A}_{e}=\bar{A}-L \bar{C}$.

In order to guarantee the effectiveness of the ESO, two assumptions should be satisfied as follows.

Assumption 2. There exists matrix $L$ such that the following equality is established:

$$
\bar{A}_{e}^{T} P+P \bar{A}_{e}=-Q
$$

where $Q, P$ are positive symmetric matrixes.

Assumption 3. For a practical MAGC system, the unknown functions $f_{1}(x, t), f_{2}(x, t)$ and their derivatives $h_{1}(t), h_{2}(t)$ are all bounded; that is, $\|f(x, t)\| \leq f_{d}$ and $\|h(t)\| \leq h_{d}$, where $f_{d}$ and $h_{d}$ are positive scalars.
First, we can get the following theorem.

Theorem 4. For system (15), design the ESO as (18). Then, the estimate error $\tilde{x}$ is uniformly ultimately bounded (UUB).

Proof. Choose the Lyapunov function as

$$
V_{0}=\tilde{x}^{T} P \tilde{x}
$$

Then, the derivative of $V$ yields

$$
\begin{aligned}
\dot{V}_{0} & =-\tilde{x}^{T} Q \tilde{x}+2 \tilde{x}^{T} P E h(t) \\
& \leq-\tilde{x}^{T} Q \tilde{x}+\frac{1}{b} \tilde{x}^{T} P P \tilde{x}+b\|E h(t)\|^{2} \\
& =-\tilde{x}^{T}\left(Q-\frac{1}{b} P P\right) \tilde{x}+b\left(h_{1}^{2}+h_{2}^{2}\right) \\
& =-\tilde{x}^{T}\left(Q-\frac{1}{b} P P\right) \tilde{x}+b h_{d}^{2} \\
& \leq-\lambda_{\min }\left(Q-\frac{1}{b} P P\right) \tilde{x}^{T} \tilde{x}+b h_{d}^{2}
\end{aligned}
$$

where $b$ is a positive constant. If we choose $b$ and $P$ to make $Q-(1 / b) P P$ positive, then $\dot{V}_{0}$ is negative as long as

$$
\|\tilde{x}\|^{2}>\frac{b h_{d}^{2}}{\lambda_{\min }(Q-(1 / b) P P)} .
$$

Therefore, $\dot{V}_{0}$ is negative outside a compact set in the $\|\widetilde{x}\|^{2}$ plane, which is thus shown to be an attractive set for the system. Based on Lyapunov stability theory, the UUB property of $\tilde{x}$ is achieved. The proof is completed.

Remark 5. Assumptions 2 and 3 are necessary conditions to design the ESO. Based on Assumption 2, we can get that there must exist positive constant $b$ to make $Q-(1 / b) P P$ positive, and the minimum eigenvalue of $Q-(1 / b) P P$ affects the size of convergence region, which is larger and better.

Remark 6. From (24), we can see that the approximation error $\tilde{x}$ is fundamentally bounded by $h_{d}$. If $h_{d}=0$, the rate of change of $f(x, t)$ is zero, and the tracking error $\tilde{x}$ will converge to zero asymptotically.

\section{The ESO Based Dynamic Surface Control}

In this section, we adopt DSC technique to design the controller. The design procedure of the DSC system contains 
4 steps similar to the backstepping design method. We choose coordinate transformation as follows:

$$
\begin{aligned}
& z_{1}=x_{1}, \\
& z_{2}=x_{2}-\alpha_{1}, \\
& s_{1}=\alpha_{1}-\alpha_{11}, \\
& z_{3}=x_{3}-\alpha_{2}, \\
& s_{2}=\alpha_{2}-\alpha_{22}, \\
& z_{4}=x_{4}-\alpha_{3}, \\
& s_{3}=\alpha_{3}-\alpha_{33} .
\end{aligned}
$$

And we pass virtual controllers $\alpha_{11}, \alpha_{22}, \alpha_{33}$ through a firstorder filter as

$$
\begin{array}{ll}
\zeta_{1} \dot{\alpha}_{1}+\alpha_{1}=\alpha_{11}, & \alpha_{1}(0)=0, \\
\zeta_{2} \dot{\alpha}_{2}+\alpha_{2}=\alpha_{22}, & \alpha_{2}(0)=0, \\
\zeta_{3} \dot{\alpha}_{3}+\alpha_{3}=\alpha_{33}, & \alpha_{3}(0)=0,
\end{array}
$$

where $\zeta_{i}, i=1,2,3$ are positive constants.

Step 1. Choose $V_{1}=(1 / 2) z_{1}^{2}$, and then the time derivative of $V_{1}$ is

$$
\begin{aligned}
\dot{V}_{1}= & z_{1}\left(-a_{11} z_{1}-a_{11} x_{2}+f_{1}\right) \\
\leq & z_{1}\left(-a_{11} z_{1}-a_{11}\left(z_{2}+s_{1}+\alpha_{11}\right)+\tilde{x}_{5}+\widehat{x}_{5}\right) \\
\leq & z_{1}\left(-a_{11} z_{1}-a_{11}\left(z_{2}+\alpha_{11}\right)\right)+\frac{b_{1}}{4} z_{1}^{2}+\frac{1}{b_{1}} \widetilde{x}_{5}^{2} \\
& +\frac{b_{2}}{4} z_{1}^{2} \widehat{x}_{5}^{2}+\frac{1}{b_{2}}+\frac{a_{11}^{2}}{2 \lambda_{1}} z_{1}^{2}+\frac{\lambda_{1}}{2} s_{1}^{2},
\end{aligned}
$$

where $b_{1}, b_{2}, \lambda_{1}$ are positive constants to be designed later.

We design the virtual controller $\alpha_{11}$ as

$$
\alpha_{11}=a_{11}^{-1}\left(-a_{11} z_{1}+\frac{b_{1}}{4} z_{1}+\frac{b_{2}}{4} z_{1} \widehat{x}_{5}^{2}+z_{1}+\frac{a_{11}^{2}}{2 \lambda_{1}} z_{1}\right) \text {. }
$$

Submmiting (28) into (27), we can get

$$
\dot{V}_{1} \leq-z_{1}^{2}-z_{1} a_{11} z_{2}+\frac{\lambda_{1}}{2} s_{1}^{2}+\frac{1}{b_{1}} \widetilde{x}_{5}^{2}+\frac{1}{b_{2}} .
$$

Step 2. Choose $V_{2}=V_{1}+(1 / 2) z_{2}^{2}$, and then the time derivative of $V_{2}$ is

$$
\begin{aligned}
\dot{V}_{2} & =\dot{V}_{1}+z_{2}\left(x_{3}-\dot{\alpha}_{1}\right) \\
& \leq \dot{V}_{1}+z_{2}\left(z_{3}+s_{2}+\alpha_{22}+\frac{s_{1}}{\zeta_{1}}\right) \\
& \leq \dot{V}_{1}+z_{2}\left(z_{3}+\alpha_{22}+\frac{s_{1}}{\zeta_{1}}\right)+\frac{1}{2 \lambda_{2}} z_{2}^{2}+\frac{\lambda_{2}}{2} s_{2}^{2},
\end{aligned}
$$

where $\lambda_{2}$ is positive constant.
We design the virtual controller $\alpha_{22}$ as

$$
\alpha_{22}=-\left(\frac{s_{1}}{\zeta_{1}}-a_{11} z_{1}+z_{2}+\frac{1}{2 \lambda_{2}} z_{2}\right) .
$$

Submmiting (31) into (30), we can get

$$
\dot{V}_{2} \leq-z_{1}^{2}-z_{2}^{2}+\frac{\lambda_{1}}{2} s_{1}^{2}+\frac{\lambda_{2}}{2} s_{2}^{2}+\frac{1}{b_{1}} \tilde{x}_{5}^{2}+\frac{1}{b_{2}}+z_{2} z_{3} .
$$

Step 3. Choose $V_{3}=V_{2}+(1 / 2) z_{3}^{2}$, and then the time derivative of $V_{3}$ is

$$
\begin{aligned}
\dot{V}_{3} & =\dot{V}_{2}+z_{3}\left(x_{4}-\dot{\alpha}_{2}\right) \\
& \leq \dot{V}_{2}+z_{3}\left(z_{4}+s_{3}+\alpha_{33}+\frac{s_{2}}{\zeta_{2}}\right) \\
& \leq \dot{V}_{2}+z_{3}\left(z_{4}+\alpha_{33}+\frac{s_{2}}{\zeta_{2}}\right)+\frac{1}{2 \lambda_{3}} z_{3}^{2}+\frac{\lambda_{3}}{2} s_{3}^{2},
\end{aligned}
$$

where $\lambda_{3}$ is positive constant.

We design the virtual controller $\alpha_{33}$ as

$$
\alpha_{33}=-\left(\frac{s_{2}}{\zeta_{2}}+z_{2}+z_{3}+\frac{1}{2 \lambda_{3}} z_{3}\right) .
$$

Submmiting (34) into (33), we can get

$$
\begin{aligned}
\dot{V}_{3} \leq & -z_{1}^{2}-z_{2}^{2}-z_{3}^{2}+\frac{1}{b_{1}} \tilde{x}_{5}^{2}+\frac{1}{b_{2}}+\frac{\lambda_{1}}{2} s_{1}^{2}+\frac{\lambda_{2}}{2} s_{2}^{2} \\
& +\frac{\lambda_{3}}{2} s_{3}^{2}+z_{3} z_{4} .
\end{aligned}
$$

Step 4. Choose $V_{4}=V_{3}+(1 / 2) z_{4}^{2}$, and then the time derivative of $V_{4}$ is

$$
\begin{aligned}
\dot{V}_{4} \leq & \dot{V}_{3}+z_{4}\left(v+g(x)+f_{2}-\dot{\alpha}_{3}\right) \\
\leq & \dot{V}_{3}+z_{4}\left(v+g(x)+\widetilde{x}_{6}+\widehat{x}_{6}+\frac{s_{3}}{\zeta_{3}}\right) \\
\leq & \dot{V}_{3}+z_{4}\left(v+g(x)+\frac{s_{3}}{\zeta_{3}}\right)+\frac{b_{11}}{4} z_{4}^{2}+\frac{b_{3}}{4} z_{4}^{2} \widehat{x}_{6}^{2} \\
& +\frac{1}{b_{3}}+\frac{1}{b_{11}} \tilde{x}_{6}^{2} .
\end{aligned}
$$

We design the controller $v$ as

$$
v=-\left(g(x)+\frac{s_{3}}{\zeta_{3}}+\frac{b_{11}}{4} z_{4}+\frac{b_{3}}{4} z_{4} \widehat{x}_{6}^{2}+z_{3}+z_{4}\right) .
$$

Submmiting (37) into (36), we can get

$$
\begin{aligned}
\dot{V}_{4} \leq & -z_{1}^{2}-z_{2}^{2}-z_{3}^{2}-z_{4}^{2}+\frac{1}{b_{1}} \tilde{x}_{5}^{2}+\frac{1}{b_{11}} \tilde{x}_{6}^{2}+\frac{1}{b_{2}}+\frac{1}{b_{3}} \\
& +\frac{\lambda_{1}}{2} s_{1}^{2}+\frac{\lambda_{2}}{2} s_{2}^{2}+\frac{\lambda_{3}}{2} s_{3}^{2} .
\end{aligned}
$$


Theorem 7. For MAGC system (11) with the control input designed as (37) and ESO (18), the estimation error $\tilde{x}(t)$ and the states are semiglobal uniformly ultimately bounded.

Proof. Choose the Lyapunov function as

$$
V=V_{0}+V_{4}+V_{5}
$$

where $V_{5}=(1 / 2) s_{1}^{2}+(1 / 2) s_{2}^{2}+(1 / 2) s_{3}^{2}$.

First, we calculate the derivative of $V_{5}$, which is

$$
\begin{aligned}
\dot{V}_{5} \leq & s_{1}\left(\dot{\alpha}_{1}-\dot{\alpha}_{11}\right)+s_{2}\left(\dot{\alpha}_{2}-\dot{\alpha}_{22}\right)+s_{3}\left(\dot{\alpha}_{3}-\dot{\alpha}_{33}\right) \\
\leq & \left(-\frac{s_{1}^{2}}{\zeta_{1}}+\frac{s_{1}^{2} \dot{\alpha}_{11}^{2}}{2 \lambda_{11}}+\frac{\lambda_{11}}{2}\right) \\
& +\left(-\frac{s_{2}^{2}}{\zeta_{2}}+\frac{s_{2}^{2} \dot{\alpha}_{22}^{2}}{2 \lambda_{22}}+\frac{\lambda_{22}}{2}\right) \\
& +\left(-\frac{s_{3}^{2}}{\zeta_{3}}+\frac{s_{3}^{2} \dot{\alpha}_{33}^{2}}{2 \lambda_{33}}+\frac{\lambda_{33}}{2}\right) .
\end{aligned}
$$

Then, the time derivative of $V$ is

$$
\begin{aligned}
\dot{V} \leq & \tilde{x}^{T}\left(\bar{A}_{e}^{T} P+P \bar{A}_{e}+\frac{1}{b} P P+\frac{1}{b_{1}} I+\frac{1}{b_{11}} I\right) \tilde{x}-z_{1}^{2} \\
& -z_{2}^{2}-z_{3}^{2}-z_{4}^{2}+s_{1}^{2}\left(-\frac{1}{\zeta_{1}}+\frac{\dot{\alpha}_{11}^{2}}{2 \lambda_{11}}+\frac{\lambda_{1}}{2}\right) \\
& +s_{2}^{2}\left(-\frac{1}{\zeta_{2}}+\frac{\dot{\alpha}_{22}^{2}}{2 \lambda_{22}}+\frac{\lambda_{2}}{2}\right) \\
& +s_{3}^{2}\left(-\frac{1}{\zeta_{3}}+\frac{\dot{\alpha}_{33}^{2}}{2 \lambda_{33}}+\frac{\lambda_{3}}{2}\right)+\Omega,
\end{aligned}
$$

where $\Omega=1 / b_{2}+1 / b_{3}+\lambda_{11} / 2+\lambda_{22} / 2+\lambda_{33} / 2+b h_{d}^{2}$ and $I$ is identity matrix.

Now we illustrate correctness of Theorem 7. First, we choose parameters $b, b_{1}, b_{11}$ such that $\left(\bar{A}_{e}^{T} P+P \bar{A}_{e}+(1 / b) P P+\right.$ $\left.\left(1 / b_{1}\right) I+\left(1 / b_{11}\right) I\right)$ is negative. Then, choosing $\zeta_{1}$ makes $\left(-1 / \zeta_{1}+\dot{\alpha}_{11}^{2} / 2 \lambda_{11}+\lambda_{1} / 2\right)$ negative for $\forall x \in \Phi$, where $\Phi=$ $\left\{x \mid z_{1}^{2}+z_{2}^{2}+z_{3}^{2}+z_{4}^{2} \leq \Omega\right\}$. The design of $\zeta_{2}, \zeta_{3}$ is similar to the design of $\zeta_{1}$. Finally the converge region will be contained in $\Phi$ and smaller than $\Phi$.

Remark 8. From $\Omega$ and controller design process, we know that positive constants $b_{2}, b_{3}, \lambda_{11}, \lambda_{22}, \lambda_{33}$ can be chosen arbitrarily, but the design of $b$ is limited, which cannot make the converge region arbitrary small. If $b h_{d}^{2}=0$ (i.e., $h_{d}=0$ ), we can design parameters such that the converge region is arbitrarily small. If $b h_{d}^{2} \neq 0$, we first choose appropriate $b$ to make $\left(\bar{A}_{e}^{T} P+P \bar{A}_{e}+(1 / b) P P+\left(1 / b_{1}\right) I+\left(1 / b_{11}\right) I\right)$ negative and design small enough $\zeta_{i}$ to make $\left(-1 / \zeta_{1}+\dot{\alpha}_{11}^{2} / 2 \lambda_{11}+\lambda_{1} / 2\right)$ negative for $\forall x \in \Phi$. Then, the states of the system will be bounded. Further, the value of $\zeta_{i}$ is smaller, and the initial region is larger, in which the function $\left(-1 / \zeta_{1}+\dot{\alpha}_{11}^{2} / 2 \lambda_{11}+\right.$ $\left.\lambda_{1} / 2\right)$ is always negative.

\section{General High-Order System}

The MAGC system we discuss is a fourth-order system, which has two unknown nonlinear terms and two outputs. The design of ESO for high-order systems has been investigated in many literatures; see $[24,25]$. In this section, we consider a class of general high-order system, which is described as follows:

$$
\begin{aligned}
& \dot{x}=A x+g(x)+B_{u} u+B_{d} f(x, t), \\
& y=C x,
\end{aligned}
$$

where $B_{d}$ is $n \times m, f(x, t)=\left[f_{1}(x, t), \ldots, f_{m}(x, t)\right]^{T}$, and $C$ satisfies (44) in Assumption 9:

$$
\begin{aligned}
A & =\left[\begin{array}{ccccc}
\bar{a}_{11} & a_{1} & 0 & \cdots & 0 \\
\vdots & 0 & \ddots & \cdots & \vdots \\
\vdots & \vdots & \vdots & \ddots & 0 \\
\vdots & \vdots & \cdots & \cdots & a_{n-1} \\
\bar{a}_{n 1} & 0 & 0 & \cdots & 0
\end{array}\right]_{n \times n}, \\
g(x) & =\left[\begin{array}{c}
g_{1}\left(x_{1}\right) \\
\vdots \\
g_{j}\left(\bar{x}_{j}\right) \\
g_{n}\left(\bar{x}_{n}\right)
\end{array}\right], \\
B_{u} & =\left[\begin{array}{l}
0 \\
\vdots \\
0 \\
1
\end{array}\right],
\end{aligned}
$$

where $a_{1}, \ldots, a_{n-1}, \bar{a}_{11}, \ldots, \bar{a}_{n 1}$ are known constants.

Assumption 9. The number of outputs is not less than $m$. Without loss of generality, we assume $C$ is as the following form:

$$
C=\left[I_{m \times m} \mid 0\right]_{m \times n},
$$

where $I$ is an identity matrix.

Assumption 10. The unknown functions $f_{1}(x, t), \ldots, f_{m}(x, t)$ and their derivatives $h_{1}(t), \ldots, h_{m}(t)$ are all bounded; that is, $\|f(x, t)\| \leq f_{d}$ and $\|h(t)\| \leq h_{d}$, where $f(x, t)=$ $\left[f_{1}(x, t), \ldots, f_{m}(x, t)\right]^{T}, h(t)=\left[h_{1}(t), \ldots, h_{m}(t)\right]^{T}$, and $f_{d}, h_{d}$ are positive scalars, which is similar to Assumption 3.

Assumption 11. The smooth function $g_{j}(\cdot)$ satisfies the Lipschitz condition, which is

$$
\left|g_{j}\left(\bar{x}_{j}(t)\right)-g_{j}\left(\overline{\hat{x}}_{j}(t)\right)\right| \leq \rho_{j}\left\|\bar{x}_{j}(t)-\overline{\hat{x}}_{j}(t)\right\|,
$$

where $\rho_{j}$ is a known positive Lipschitz constant; $\bar{x}_{j}=$ $\left[x_{1}, \ldots, x_{j}\right]^{T}, \overline{\widehat{x}}_{j}=\left[\widehat{x}_{1}, \ldots, \widehat{x}_{j}\right]^{T}$. 
Then, the extended state equation is given by

$$
\begin{aligned}
& \dot{\bar{x}}=\bar{A} \bar{x}+\bar{B}_{u} u+E h(t), \\
& y=\bar{C} \bar{x},
\end{aligned}
$$

where variables

$$
\begin{aligned}
\bar{x} & =\left[\begin{array}{llll}
x & x_{n+1} & \cdots & x_{n+m}
\end{array}\right]^{T}, \\
h(t) & =\left[\begin{array}{lll}
h_{1} & \cdots & h_{m}
\end{array}\right]^{T}
\end{aligned}
$$

and matrices

$$
\begin{aligned}
\bar{A} & =\left[\begin{array}{cc}
A_{n \times n} & \left(B_{d}\right)_{n \times m} \\
0_{m \times n} & 0_{m \times m}
\end{array}\right], \\
\bar{B}_{u} & =\left[\begin{array}{c}
\left(b_{u}\right)_{n \times 1} \\
0_{m \times 1}
\end{array}\right], \\
E & =\left[\begin{array}{c}
0_{n \times m} \\
I_{m \times m}
\end{array}\right], \\
\bar{C} & =\left[\begin{array}{ll}
C_{m \times n} & 0_{m \times m}
\end{array}\right] .
\end{aligned}
$$

For system (46), we design the ESO as follows:

$$
\dot{\hat{x}}=\bar{A} \hat{x}+\bar{B}_{u} u+\widehat{g}(\widehat{x})+L(y-\widehat{y}), \quad \widehat{y}=\bar{C} \hat{x},
$$

where $\hat{x}$ is the estimate of the state $\bar{x}$ and $L$ is the observer gain matrix with dimension $(m+n) \times m$. Consider $\widehat{g}(\widehat{x})=$ $\left[\widehat{g}_{1}\left(\widehat{x}_{1}\right), \ldots, \widehat{g}_{n}\left(\bar{x}_{n}\right), 0_{1 \times m}\right]^{T}$.

The extended state error is defined as

$$
\tilde{x}=\bar{x}-\widehat{x} .
$$

Subtracting (49) from (46), we get

$$
\dot{\tilde{x}}=\bar{A}_{e} \tilde{x}+E h(t),
$$

where $\bar{A}_{e}=\bar{A}-L \bar{C}$.

In order to guarantee the effectiveness of the ESO, the following assumption should be satisfied.

Assumption 12. There exists matrix $L$ such that the following equality is established:

$$
\bar{A}_{e}^{T} P+P \bar{A}_{e}+b P P+\bar{b} P P+\frac{1}{\bar{b}} \rho=-Q,
$$

where $Q, P$ are positive symmetric matrixes and $b, \bar{b}$ are positive constants, $\rho=\sum_{j=1}^{n} \rho_{j}^{2}$.

Next, we can get the following theorem.

Theorem 13. For system (42), design the ESO as (49). Then, the estimate error $\tilde{x}$ is uniformly ultimately bounded (UUB).

Proof. Define the Lyapunov function as

$$
V_{0}=\tilde{x}^{T} P \widetilde{x} .
$$

The derivative of $V_{0}$ yields

$$
\begin{aligned}
\dot{V}_{0}= & -\tilde{x}^{T}\left(\bar{A}_{e}^{T} P+P \bar{A}_{e}\right) \tilde{x}+\tilde{x}^{T} P E h(t) \\
& +\tilde{x}^{T}(g(x)-\hat{g}(\hat{x})) \\
\leq & \tilde{x}^{T}\left(\bar{A}_{e}^{T} P+P \bar{A}_{e}+\frac{1}{b} P P+\bar{b} P P+\frac{1}{\bar{b}} \rho\right) \tilde{x}+b h_{d}^{2} .
\end{aligned}
$$

Then $\dot{V}_{0}$ is negative as long as

$$
\|\tilde{x}\|^{2}>\frac{b h_{d}^{2}}{\lambda_{\min } Q} .
$$

The proof is completed.

Next, we can design the controller using (49) by DSC. The coordinate transformation can be chosen as follows:

$$
\begin{aligned}
& z_{1}=\widehat{x}_{1}, \\
& z_{2}=\widehat{x}_{2}-\alpha_{1}, \\
& s_{1}=\alpha_{1}-\alpha_{11}, \\
& z_{j}=\widehat{x}_{j}-\alpha_{j}, \\
& s_{j}=\alpha_{j}-\alpha_{j j}, \\
& z_{n}=\widehat{x}_{n}-\alpha_{n}, \\
& s_{n}=\alpha_{n}-\alpha_{n n} .
\end{aligned}
$$

The design process is similar to the previous section and thus omitted. Similar theorem also can be obtained.

Remark 14. In Assumption 9, we assume that the number of outputs is not less than $m$, which is a necessary condition to guarantee Assumption 12 to be established. Assumption 11 is a common assumption to design observer in nonlinear systems, and $g(x)$ in (12) also satisfies this condition.

Remark 15. From (56), we know that $\hat{x}$ is used to design the controller, so $x$ need not be measurable. In the previous section, we do not use this kind of method, because Assumption 12 is more conservative than Assumption 2 and in MAGC systems the parameters values are very large such that calculating better design parameters is difficult to meet the demand of engineering, which can be seen in the following simulation.

\section{Simulations}

6.1. MAGC System. In order to verify the effectiveness of the proposed strategy, we make simulations for a $1700 \mathrm{~mm}$ rolling mill system. The technical parameters of rolling mill are as follows: supporting roll $\phi 1371 \times 1700 \mathrm{~mm}$ and working roll $\phi 914 \times 1700 \mathrm{~mm}$. The hydraulic system parameters are as follows: cylinder diameter $\phi 720 \mathrm{~mm}$, stem diameter $\phi 600 \mathrm{~mm}$, and stroke $40 \mathrm{~mm}$. The obtained main parameters of the system are shown in Table 1. 
TABLE 1: Main parameters in the MAGC system.

\begin{tabular}{lc}
\hline Prm & Value \\
\hline$K_{p}$ & $0.002 \mathrm{~A} / \mathrm{V}$ \\
$\omega$ & $6.283 \times 10^{-2} \mathrm{~m}$ \\
$\omega_{v}$ & $628 \mathrm{rad} / \mathrm{s}$ \\
$\beta_{e}$ & $800 \mathrm{MPa}$ \\
$C_{t}$ & $2.4 \times 10^{-12} \mathrm{~m}^{3} / \mathrm{Pa} \cdot \mathrm{s}$ \\
$M_{t}$ & $2.36 \times 10^{5} \mathrm{~kg}$ \\
$K_{t}$ & $5.3 \times 10^{9} \mathrm{~N} / \mathrm{m}$ \\
$T_{h}$ & $0.125 \mathrm{~s}$ \\
$\zeta_{v}$ & 0.7 \\
$\rho$ & $900 \mathrm{~kg} / \mathrm{m}^{3}$ \\
$K_{v}$ & $0.02 \mathrm{~A} / \mathrm{m}$ \\
$A_{p}$ & $0.41 \mathrm{~m}{ }^{2}$ \\
$V$ & $6.39 \times 10^{-3} \mathrm{~m}^{3}$ \\
$B_{p}$ & $3.64 \times 10^{7} \mathrm{~N} \cdot \mathrm{s} / \mathrm{m}$ \\
$W_{s}$ & $9.434 \times 10^{9} \mathrm{~N} / \mathrm{m}$ \\
$T$ & $0.5 \mathrm{~s}$ \\
\hline
\end{tabular}

The desired exit thickness $h_{\text {out } d}$ is $1 \mathrm{~mm}$, and desired cylinder piston displacement $x_{p d}$ is $1 \mathrm{~mm}$. The state variables are errors between desired values and practical ones. So the state should converge to zero with an acceptable accuracy.

Using LMI, we can design parameters to make $\left(\bar{A}_{e}^{T} P+\right.$ $\left.P \bar{A}_{e}+(1 / b) P P+\left(1 / b_{1}\right) I+\left(1 / b_{11}\right) I\right)$ negative. The designed parameters are as follows: $Q=0.0046 I, b=0.2366, b_{1}=$ $b_{11}=76.2361, b_{2}=b_{3}=10, \lambda_{1}=10, \lambda_{2}=0.2, \lambda_{3}=0.2$, $\zeta_{1}=\zeta_{2}=\zeta_{3}=0.05$,

$$
L=\left[\begin{array}{cc}
10.1787 & -5.3484 \\
-3.7879 & 34.2221 \\
-34.8146 & 319.3424 \\
-54.8886 & 498.1011 \\
30.1321 & 4.2610 \\
-28.7435 & 260.5028
\end{array}\right] .
$$

Initial values are chosen as $x=10^{-3}[0.8,1,1,3]^{T}$ and $\hat{x}=10^{-3}[2,2,1,2,0,0]^{T}$. The simulation results are shown in Figures 2-9.

From Figures 2-9, we can see that the ESO can estimate the real state variables very well, and the real state and estimated state both are stabilized quickly with an acceptable accuracy.

\section{Conclusions}

The MAGC system has mismatched uncertainties and disturbances. To deal with the control problem, we present an ESO based dynamic surface control strategy. The ESO is designed at first. Then, via DSC method, the controller is designed based on constructed ESO. Based on Lyapunov stability theory, we show that the solutions converge to an adjustable region. What is more, general high-order system case is also

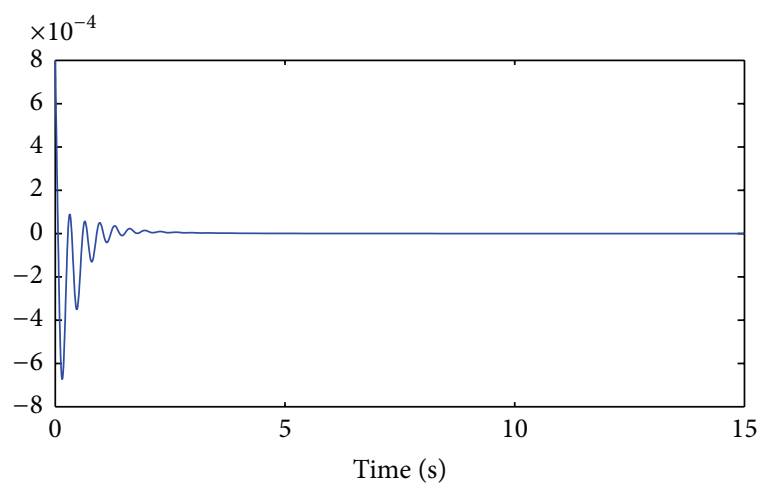

Figure 2: The response of state $x_{1}$.

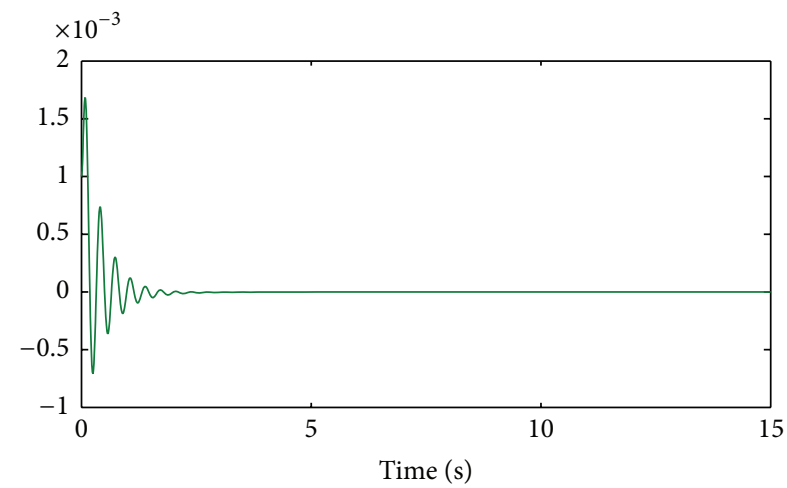

FIGURE 3: The response of state $x_{2}$.

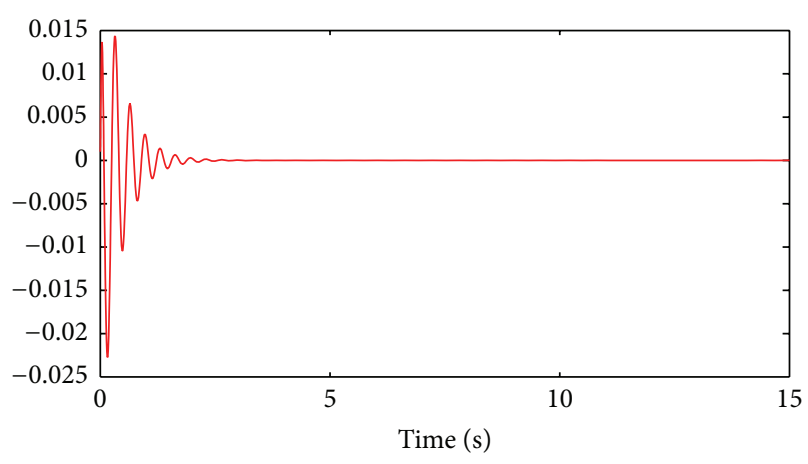

FIGURE 4: The response of state $x_{3}$.

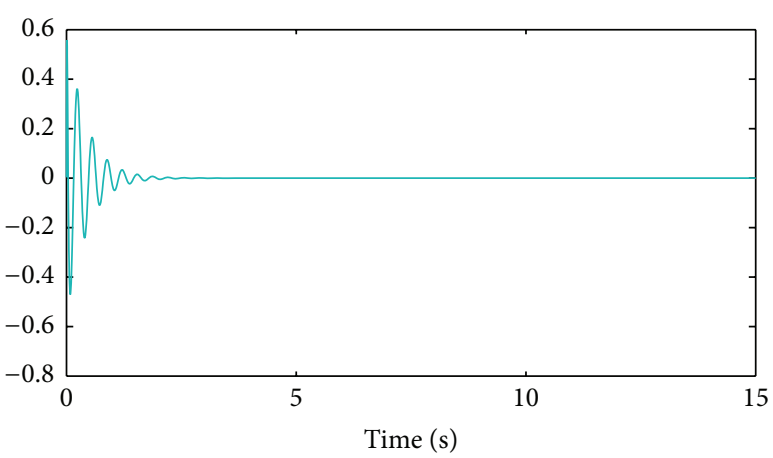

FIGURE 5: The response of state $x_{4}$. 


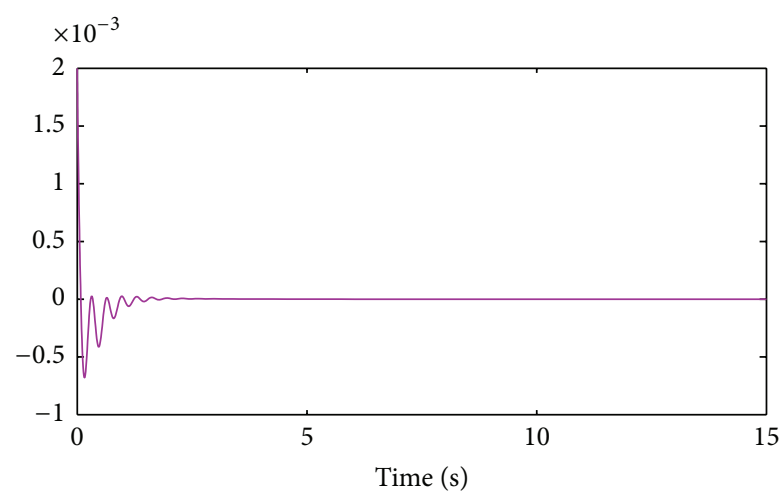

FIgURE 6: The response of estimated state $\hat{x}_{1}$.

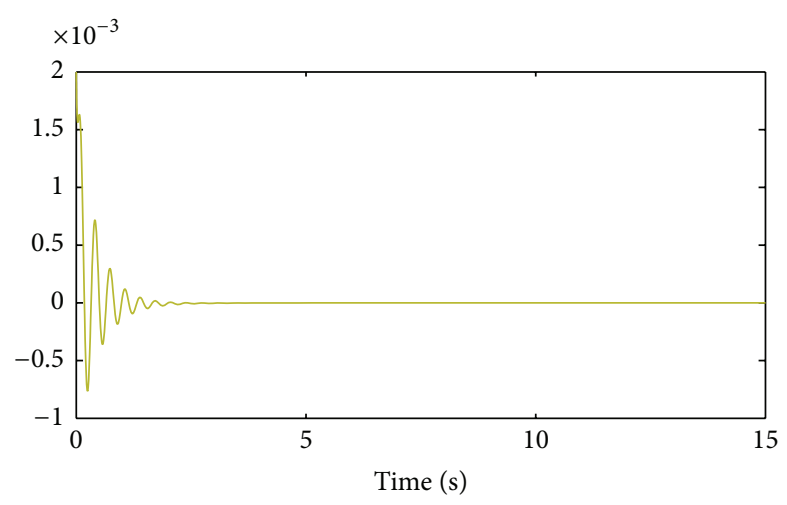

Figure 7: The response of estimated state $\widehat{x}_{2}$.

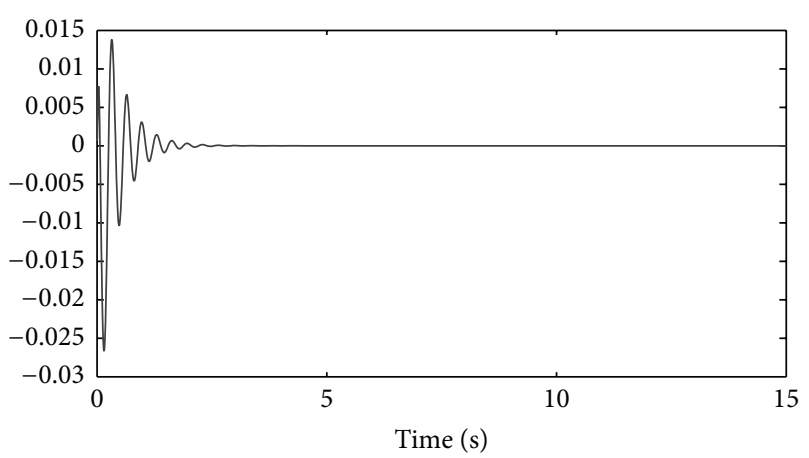

Figure 8: The response of estimated state $\hat{x}_{3}$.

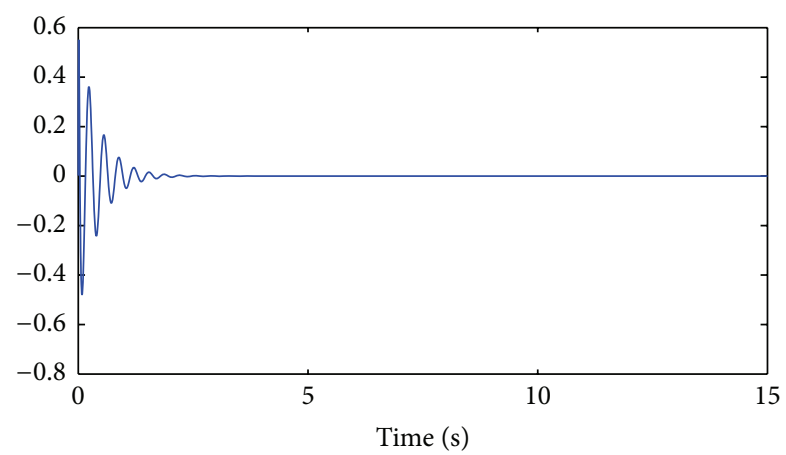

Figure 9: The response of estimated state $\hat{x}_{4}$. discussed and the corresponding control scheme is proposed. Finally, the simulation on MAGC system is performed and the results show the effectiveness of the proposed method.

\section{Conflict of Interests}

The authors declare that there is no conflict of interests regarding the publication of this paper.

\section{References}

[1] W. Soszyński and A. Studnicka, "A review of contemporary solutions for cold rolling that allow quality improvement," Journal of Achievements in Materials and Manufacturing Engineering, vol. 55, no. 2, pp. 810-816, 2012.

[2] X.-Y. Xu, Z.-W. Bu, Y.-L. Cai, and X.-Y. Xu, "Fuzzy logic based thickness control system and its simulation," in Proceedings of the 1st International Conference on Information Science and Engineering (ICISE '09), pp. 3792-3795, Nanjing, China, December 2009.

[3] C. C. Hua and C. Yu, "Controller design for cold rolling mill HAGC system with measurement delay perturbation," Journal of Mechanical Engineering, vol. 50, no. 20, pp. 46-53, 2014.

[4] Z. Gang, Y. Yuan, and L. Haowen, "Research on modeling of Hydraulic rolling mill," in Proceedings of the Chinese Control and Decision Conference (CCDC '11), pp. 1814-1818, IEEE, Mianyang, China, May 2011.

[5] S. H. Li, J. Yang, W.-H. Chen, and X. S. Chen, "Generalized extended state observer based control for systems with mismatched uncertainties," IEEE Transactions on Industrial Electronics, vol. 59, no. 12, pp. 4792-4802, 2012.

[6] S. N. Huang, K. K. Tan, and T. H. Lee, "Further result on a dynamic recurrent neural-network-based adaptive observer for a class of nonlinear systems," Automatica, vol. 41, no. 12, pp. 2161-2162, 2005.

[7] X. W. Dai, Z. W. Gao, T. Breikin, and H. Wang, "High-gain observer-based estimation of parameter variations with delay alignment," IEEE Transactions on Automatic Control, vol. 57, no. 3, pp. 726-732, 2012.

[8] K. C. Veluvolu and D. Lee, "Sliding mode high-gain observers for a class of uncertain nonlinear systems," Applied Mathematics Letters, vol. 24, no. 3, pp. 329-334, 2011.

[9] A. A. Godbole, J. P. Kolhe, and S. E. Talole, "Performance analysis of generalized extended state observer in tackling sinusoidal disturbances," IEEE Transactions on Control Systems Technology, vol. 21, no. 6, pp. 2212-2223, 2013.

[10] Z. Zhu, D. Xu, J. Liu, and Y. Xia, "Missile guidance law based on extended state observer," IEEE Transactions on Industrial Electronics, vol. 60, no. 12, pp. 5882-5891, 2013.

[11] H. Liu and S. Li, "Speed control for PMSM servo system using predictive functional control and extended state observer," IEEE Transactions on Industrial Electronics, vol. 59, no. 2, pp. 11711183, 2012.

[12] X. Shi and S. Chang, "Extended state observer-based timeoptimal control for fast and precise point-to-point motions driven by a novel electromagnetic linear actuator," Mechatronics, vol. 23, no. 4, pp. 445-451, 2013.

[13] S. C. Tong, Y. M. Li, and P. Shi, "Observer-based adaptive fuzzy backstepping output feedback control of uncertain MIMO pure-feedback nonlinear systems," IEEE Transactions on Fuzzy Systems, vol. 20, no. 4, pp. 771-785, 2012. 
[14] C. Hua, P. X. Liu, and X. Guan, "Backstepping control for nonlinear systems with time delays and applications to chemical reactor systems," IEEE Transactions on Industrial Electronics, vol. 56, no. 9, pp. 3723-3732, 2009.

[15] C. Hua and Y. Li, "Output feedback prescribed performance control for interconnected time-delay systems with unknown Prandtl-Ishlinskii hysteresis," Journal of the Franklin Institute, vol. 352, no. 7, pp. 2750-2764, 2015.

[16] C. C. Hua, G. Liu, L. Zhang, and X. Guan, "Output feedback tracking control for nonlinear time-delay systems with tracking errors and input constraints," Neurocomputing, vol. 173, pp. 751758, 2016.

[17] D. Swaroop, J. K. Hedrick, P. P. Yip, and J. Gerdes, "Dynamic surface control for a class of nonlinear systems," IEEE Transactions on Automatic Control, vol. 45, no. 10, pp. 1893-1899, 2000.

[18] N. Qaiser, N. Iqbal, A. Hussain, and N. Qaiser, "Exponential stabilization of a class of underactuated mechanical systems using dynamic surface control," International Journal of Control, Automation and Systems, vol. 5, no. 5, pp. 547-558, 2007.

[19] X. Xia and T. Zhang, "Adaptive output feedback dynamic surface control of nonlinear systems with unmodeled dynamics and unknown high-frequency gain sign," Neurocomputing, vol. 143, no. 2, pp. 312-321, 2014.

[20] B. Song, "Robust stabilization of decentralized dynamic surface control for a class of interconnected nonlinear systems," International Journal of Control, Automation and Systems, vol. 5, no. 2, pp. 138-146, 2007.

[21] J. B. Park, S. J. Yoo, and Y. H. Choi, "Output feedback dynamic surface control of flexible-joint robots," International Journal of Control, Automation and Systems, vol. 6, no. 2, pp. 223-233, 2008.

[22] S. J. Yoo, J. B. Park, and Y. H. Choi, "Adaptive dynamic surface control for stabilization of parametric strict-feedback nonlinear systems with unknown time delays," IEEE Transactions on Automatic Control, vol. 52, no. 12, pp. 2360-2365, 2007.

[23] Y. Cui, H. Zhang, Y. Wang, and Z. Zhang, "Adaptive neural dynamic surface control for a class of uncertain nonlinear systems with disturbances," Neurocomputing, vol. 165, pp. 152158, 2015.

[24] M. H. Sun, W. Zhang, and Y. Q. Wang, "Modeling and simulation on screw-down electric-hydraulic servo system of cold rolling mill based on virtual rolling," Machine Tool \& Hydraulics, vol. 40, no. 9, pp. 144-146, 2012.

[25] X. Yang and Y. Huang, "Capabilities of extended state observer for estimating uncertainties," in Proceedings of the American Control Conference (ACC '09), pp. 3700-3705, IEEE, St. Louis, Mo, USA, June 2009. 


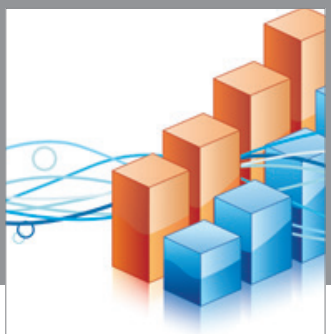

Advances in

Operations Research

vatem alat4

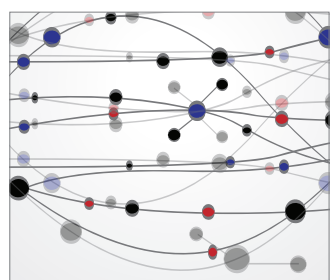

\section{The Scientific} World Journal
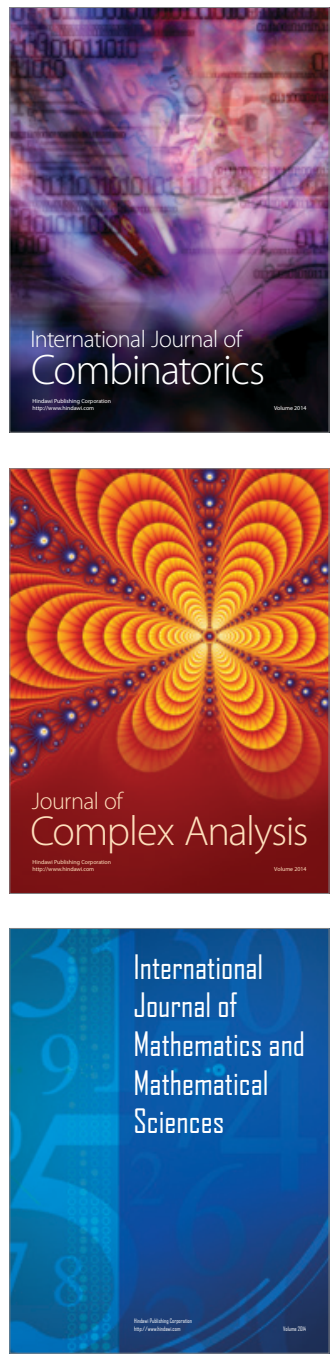
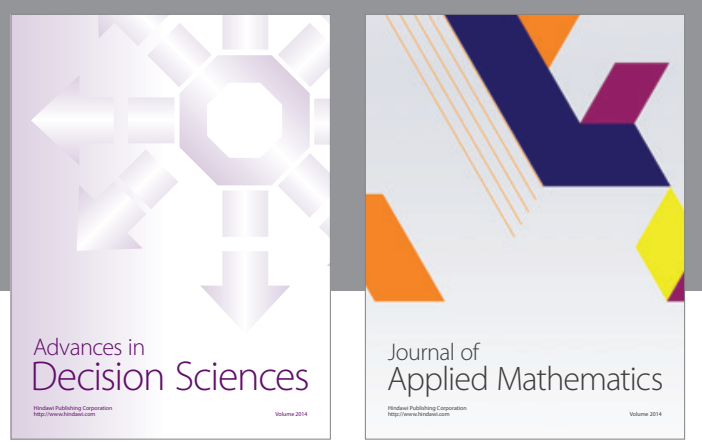

Algebra

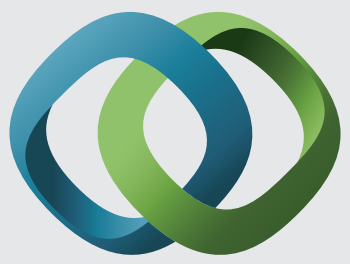

\section{Hindawi}

Submit your manuscripts at

http://www.hindawi.com
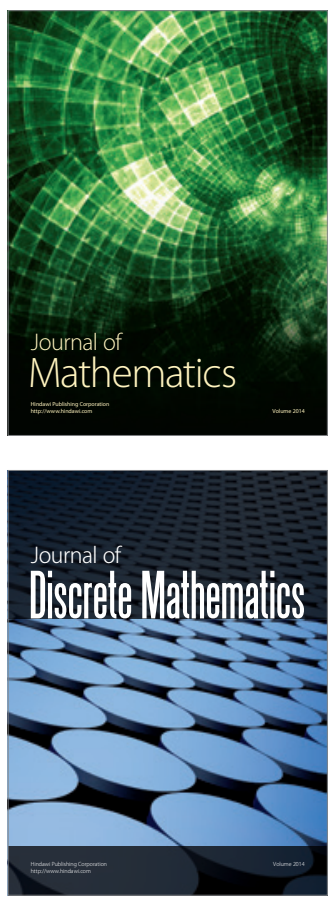

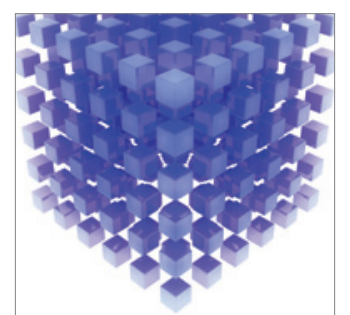

Mathematical Problems in Engineering
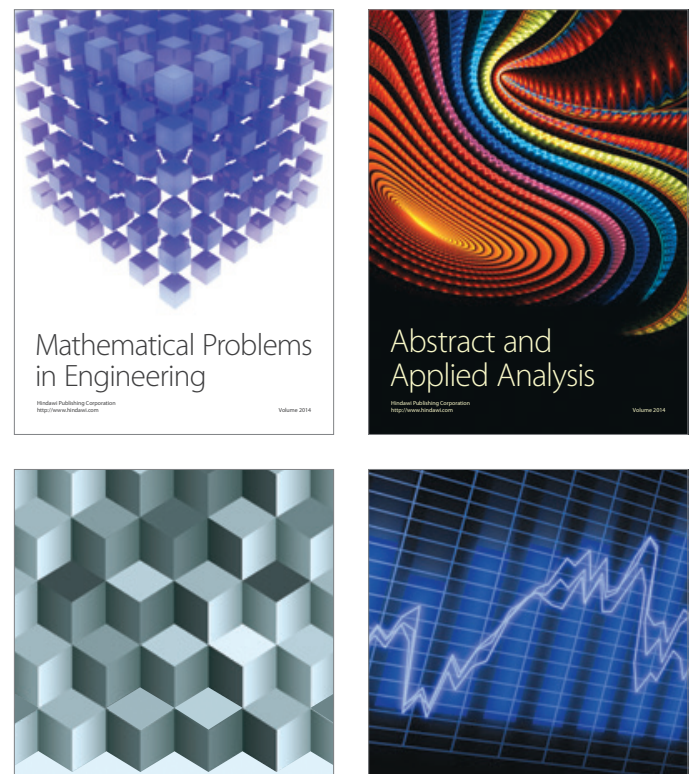

Journal of

Function Spaces

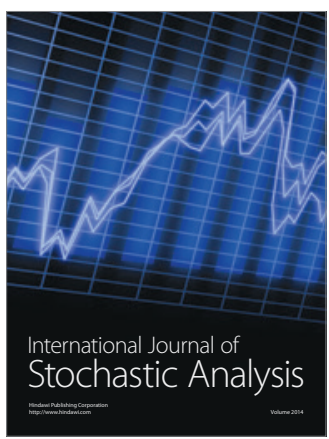

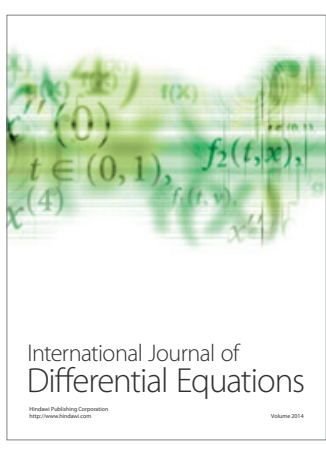
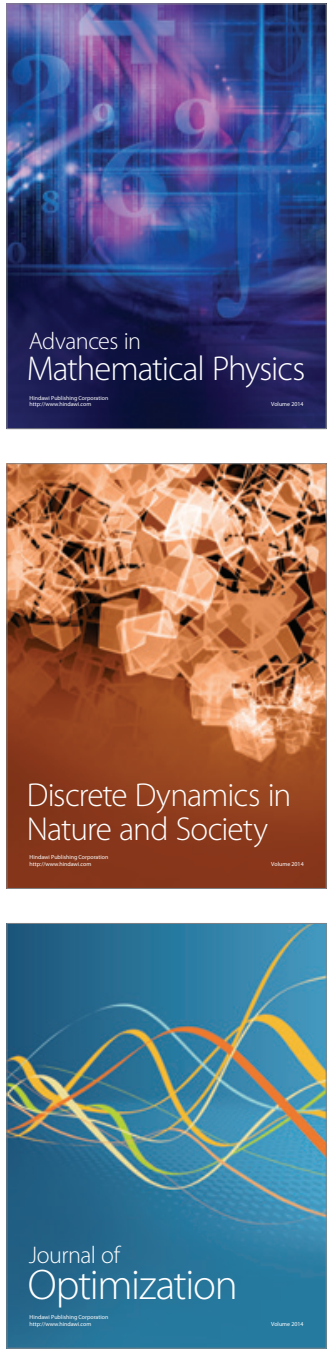